\title{
The rise of integrated quantum photonics
}

\author{
Integrated quantum photonic chips offer the promise of a convenient, scalable platform for performing tasks such \\ as quantum communication and information processing.
}

W hile research into quantum photonics has a long and rich history, in the past few decades efforts have become increasingly focused on potential applications and their practical implementation. By employing the unusual characteristics of quantum mechanics, it has long been hoped that quantum photonics could surpass the limits of the current technology. For example, quantum cryptography would make eavesdropping impossible and support perfectly secure communication while boson sampling could make quantum computation more efficient than classical processors for certain tasks. These benefits have been explored in scientific experiments and the principles are known. The big challenge is how to realize a scalable, convenient platform for practical deployment and commercialization.

Just as microelectronics transformed the modern world through the creation of the integrated circuit, which is now at the heart of most electronic devices, quantum photonics needs an equivalent platform to fulfil its application potential. In this special focus issue of Nature Photonics, we report on the progress in making this a reality with the developments in integrated quantum photonics (IQP).

In a Review Article, Jianwei Wang and colleagues provide a general overview and introduction to IQP circuits and summarize the present development of quantum hardware based on IQP chips. They remark that the challenge for measurement-based quantum computation may shift from the need for deterministic gates to constructing a generic entangled cluster-state, on which any quantum computation could be mapped by a sequence of measurements.

IQP circuits are also a desirable platform for chip-based quantum communications. However, fully integrated chip-based quantum communication has not yet been realized, largely because of the integration difficulties between silicon wafers that feature optical waveguides and other passive components and light sources and photodetectors that are made from different semiconductors. Key components such as transmitters and receivers for quantum

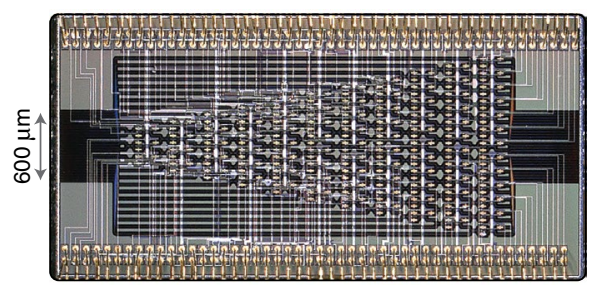

Programmable nanophotonic processor. Figure reproduced with permission from N. C. Harris et al., Nat. Photon. 11, 447-452 (2017), Springer Nature Ltd.

key distribution and quantum random number generators are instead individually fabricated.

That said, the manufacture of wafer-scale photonic circuits in silicon has undergone dramatic improvements. Like Moore's law, which forecasts a doubling in transistor count on an integrated circuit every two years, the number of integrated photonic components in an optical chip has increased exponentially from 2012 to 2019. From this fact, Wang and colleagues anticipate that very-large-scale integration of quantum photonic circuitry containing millions of components will be possible in the near future.

It is preferable for reasons of cost and convenience to fabricate IQP chips from a single material system and achieve monolithic integration. However, there is no ideal material that simultaneously delivers the required performance for all optical components such as light sources, arithmetic elements and photon detectors. As a result, it is more realistic to fabricate devices by assembling optical components made with different material systems, that is, hybrid integrated quantum photonic (HIQP) devices. In another Review Article, Ali Elshaari and colleagues summarize the progress of HIQP integration and discuss important design considerations including optical connectivity.

Integrating a suitable light source and photodetector onto a chip is an ongoing challenge. When a light source is integrated, one has to take care of heat dissipation. To date, the technique of wafer bonding
- bringing together wafers of different semiconductor materials, typically $\mathrm{Si}$ and $\mathrm{InP}-$ is proving popular. The coupling loss between an InP laser chip and a Si chip is $0.4 \mathrm{~dB}$. The wire bonding technique enables flexible hybrid integration of the best-known devices, which leads to an increase in the production yield of HIQP devices.

As for photodetectors, the high detection efficiency, low dark count rate and high timing resolution of superconducting nanowire single-photon detectors operating at cryogenic temperature makes them indispensable to measure quantum states of light. However, the issue of operation temperature is still controversial.

Because of compatibility with conventional CMOS fabrication processes, $\mathrm{SiC}$ is seen as a key material for IQP chips. Unlike Si, SiC exhibits second-order optical nonlinearity and can generate single photons from a Si vacancy. In an Article also in this issue, Daniil Lukin and colleagues report single-photon emission and second-harmonic generation from SiC-based IQP chips. It was not a straightforward task to achieve this in IQP chips because of the following two reasons: firstly, the phase matching between the fundamental and second-harmonic waves by domain-inversion control is not applicable to $\mathrm{SiC}$; and secondly, single-photon emission is overwhelmed by the strong background noise caused by the poor crystalline quality of SiC. Lukin and colleagues describe the wafer bonding and thinning technique to maintain the high crystalline quality of the $\mathrm{SiC}$ wafer, the design of high- $Q$ photonic crystal cavities to enhance single-photon emission, and the design of a high- $Q$ microring resonator to generate second-harmonic waves by modal phase-matching. This work provides a viable route towards scalable IQP chips.

The above articles indicate that progress is being made, and Nature Photonics hopes that this focus issue will help to further advance the study of IQP.

Published online: 28 April 2020 https://doi.org/10.1038/s41566-020-0634-9 